\title{
Analytical calculation of the orbital spectrum of the guiding centre motion in axisymmetric magnetic fields
}

\author{
Yannis Antonenas ${ }^{\circledR 1}$, Giorgos Anastassiou ${ }^{2}$ and Yannis Kominis ${ }^{\circledR 1}{ }^{, \dagger}$ \\ ${ }^{1}$ School of Applied Mathematical and Physical Sciences, National Technical University of Athens, \\ Athens, Greece \\ ${ }^{2}$ School of Electrical and Computer Engineering, National Technical University of Athens, Athens, Greece
}

(Received 15 August 2020; revised 18 November 2020; accepted 19 November 2020)

Charged particle motion in axisymmetric toroidal magnetic fields is analysed within the context of the canonical Hamiltonian guiding centre theory. A canonical transformation to variables measuring the drift orbit deviation from a magnetic field line is introduced and an analytical transformation to action-angle variables is obtained, under a zero drift width approximation. The latter is used to provide compact formulas for the orbital spectrum of the drift motion, namely the bounce/transit frequencies as well as the bounce/transit averaged toroidal precession and gyration frequencies. These formulas are shown to have a remarkable agreement with numerically calculated full drift width frequencies and significant differences from standard analytical formulas based on a pendulum-like Hamiltonian description. The analytical knowledge of the orbital spectrum is crucial for the formulation of particle resonance conditions with symmetry-breaking perturbations and the study of the resulting particle, energy and momentum transport.

Key words: fusion plasma, plasma dynamics, plasma nonlinear phenomena

\section{Introduction}

Charged particle dynamics in toroidal magnetic fields has been the key theoretical issue for the study of magnetically confined fusion plasmas for many decades. The understanding of the role of the magnetic field topology in single as well as collective particle dynamics has been crucial for the design of fusion devices with magnetic fields having different types of symmetries (Freidberg 2007). These background magnetic fields also determine the interactions between particles and symmetry-breaking perturbations of a large range of spatial and temporal scales, mainly through resonance conditions, and the corresponding particle, energy and momentum transport.

The guiding centre (GC) theory (Littlejohn 1983) has been used for a rigorous dynamical reduction by the systematic elimination of the rapidly varying gyro-angle variable related to the cyclotron motion around a magnetic field line. In the GC description,

$†$ Email address for correspondence: gkomin@ central.ntua.gr 
the magnetic moment is a constant of the motion and the particle dynamics is studied in terms of the drift motion of the centre of the cyclotronic motion, leading to a gyro-kinetic Hamiltonian theory. Although the original derivation of the GC equations has been formulated in non-canonical variables, the utilization of magnetic coordinates has been shown to allow a Hamiltonian formulation in canonical variables (White \& Chance 1984; Littlejohn 1985; White 2014).

The Hamiltonian formulation of the GC motion in canonical variables has several computational and conceptual advantages that are revealed under a transformation to action-angle variables (Lichtenberg \& Lieberman 1992; Goldstein, Poole \& Safko 2002). Such a transformation is possible for the case of GC motion in an axisymmetric magnetic field where the Hamiltonian system is integrable. In the action-angle variable set, the topology of the motion is described by multidimensional tori and each orbit is labelled by a distinct set of the invariant values of the three action variables (Kaufman 1972). This simple orbit parametrization allows for an orbit-based analysis of particle, energy and momentum transport which is particularly useful for the study of energetic particle dynamics in fusion plasmas in direct relation to velocity-space tomography techniques (Stagner \& Heidbrink 2017; Tholerus, Johnson \& Hellsten 2017). Moreover, all the orbital frequencies can be readily calculated in terms of the action variables. The latter determine the resonance conditions between particles and symmetry-breaking perturbations (Bierwage \& Shinohara 2016; Zestanakis et al. 2016) resulting in breaking of the action invariance and diffusion in the action space, which describes energy, momentum and radial particle transport (Kominis 2008; Kominis, Ram \& Hizanidis 2008, 2010; Petrov \& Harvey 2016). The collective particle dynamics in the presence of perturbations is characterized by the modification of the unperturbed particle distribution functions (White 2011, 2012; Podesta, Gorelenkova \& White 2014). Another important feature of the action-angle description is that the different time scales of the motion are well separated in different degrees of freedom allowing for a systematic dynamical reduction to a hierarchy of evolution equations for the reduced distribution functions (Brizard 2000; Kominis et al. 2010).

In this work, we utilize the canonical Hamiltonian formulation of the GC motion along with an appropriate canonical transformation in order to facilitate the calculation of action-angle variables for the case of an axisymmetric magnetic field. By doing so we fully exploit the advantages of the canonical Hamiltonian formulation in terms of the calculation of the orbital frequencies of all degrees of freedom corresponding to bounce/transit, bounce-averaged toroidal precession and gyration frequencies, as well as the calculation of the action variables allowing for the dynamical reduction to a bounce-averaged system. Analytical results are obtained under a zero drift width (ZDW) approximation providing compact formulas for the orbital frequencies that are in remarkable agreement with full drift width (FDW) numerical calculations and have significant qualitative and quantitative differences from standard analytical formulas based on a pendulum-like GC Hamiltonian.

In $\S 2$, the canonical formulation of the GC theory is briefly presented for completeness. In $\S 3$, we introduce a canonical transformation to variables measuring drift orbit deviation from a given field line. The transformation is general and applies to both axisymmetric and non-axisymmetric equilibria of arbitrary shape. We show that, for a large-aspect-ratio (LAR) magnetic field equilibrium, the transformation takes a particularly simple form and we briefly comment on possibilities of treating equilibria with higher-order terms with respect to the inverse aspect ratio. The general form of the transformation to action-angle variables as well as the corresponding calculation of the orbital frequencies is also presented. In $\S 4$, we apply a ZDW approximation for the LAR equilibrium, according to which the GC orbit is considered to take place on a single flux surface, and we obtain 
simple analytical formulas for the orbital spectrum of the GC motion. We present a novel ZDW Hamiltonian retaining terms that are significant for particles with smaller pitch angles in comparison to standard pendulum-like Hamiltonians describing deeply trapped particles, and we compare the analytical results to FDW numerical calculations. A summary and conclusions are given in $\S 5$.

\section{Canonical GC Hamiltonian for an axisymmetric equilibrium}

A general axisymmetric toroidal magnetic configuration consisting of nested toroidal flux surfaces can be represented in White-Boozer (White 2014) coordinates as

$$
\boldsymbol{B}=g(\psi) \nabla \zeta+I(\psi) \nabla \theta+\delta(\psi, \theta) \nabla \psi_{p}
$$

where $\zeta$ and $\theta$ are the toroidal and poloidal angles. The toroidal flux $\psi$ is related to the poloidal flux $\psi_{p}$ through the safety factor $q(\psi)=\mathrm{d} \psi / \mathrm{d} \psi_{p}$. The functions $g$ and $I$ are related to the poloidal and toroidal currents and $\delta$ is related to the non-orthogonality of the coordinate system.

The GC motion of a charged particle is described by the Lagrangian (Littlejohn 1983) $L=\left(\boldsymbol{A}+\rho_{\|} \boldsymbol{B}\right) \cdot \boldsymbol{v}+\mu \dot{\xi}-H$, where $\boldsymbol{A}$ and $\boldsymbol{B}$ are the vector potential and the magnetic field, $\boldsymbol{v}$ is the GC velocity, $\mu$ is the magnetic moment, $\xi$ is the gyro-phase, $\rho_{\|}$is the velocity component parallel to the magnetic field, normalized to $B$, and

$$
H=\rho_{\|}^{2} B^{2} / 2+\mu B
$$

is the Hamiltonian. The GC motion is given in normalized units where time is normalized to $\omega_{0}^{-1}$, with $\omega_{0}=e B_{0} / m$ being the on-axis gyro-frequency, and distance is normalized to the major radius $R$, so that energy is normalized to $m \omega_{0}^{2} R^{2}$. According to the ordering of the GC approximation, the gyro-radius is $\rho=v / B<<1$ and the magnetic moment $\mu=v_{\perp}^{2} /(2 B)$ as well as the cross-field drift are of order $\rho^{2}$ (White 2014).

The three couples of canonically conjugate variables for this GC Hamiltonian are $(\mu, \xi)$, $\left(P_{\theta}, \theta\right)$ and $\left(P_{\zeta}, \zeta\right)$ with

$$
\left.\begin{array}{l}
P_{\theta}=\psi+\rho_{\|} I(\psi) \\
P_{\zeta}=\rho_{\|} g(\psi)-\psi_{p}(\psi)
\end{array}\right\}
$$

providing the relation between the canonical momenta $\left(P_{\theta}, P_{\zeta}\right)$ and $\left(\psi, \rho_{\|}\right)$(White 2014). In terms of these canonical variables, (2.2) can be written as

$$
H\left(P_{\theta}, \theta, P_{\zeta}, \zeta, \mu, \xi\right)=\frac{\left[P_{\zeta}+\psi_{p}\left(P_{\theta}, P_{\zeta}\right)\right]^{2}}{2 g^{2}\left(\psi\left(P_{\theta}, P_{\zeta}\right)\right)} B^{2}\left(\psi\left(P_{\theta}, P_{\zeta}\right), \theta\right)+\mu B\left(\psi\left(P_{\theta}, P_{\zeta}\right), \theta\right)
$$

The gyro-angle $\xi$ does not appear in the GC Hamiltonian which is also independent of $\zeta$ due to axisymmetry of the magnetic field; therefore the corresponding canonical momenta, namely $\mu$ and $P_{\zeta}$, are constants of the motion and since the Hamiltonian does not depend explicitly on time (autonomous system), the system is integrable.

\section{Canonical transformation to drift orbit deviation variables}

The GC Hamiltonian describes all particle drifts due to the inhomogeneity of the magnetic field, causing the GC deviation from a field line. A canonical transformation 
with generating function (Goldstein et al. 2002)

$$
\bar{F}\left(P_{\theta}, \bar{\theta}, P_{\zeta}, \bar{\zeta}, \mu, \bar{\xi}\right)=-\bar{\theta}\left(P_{\theta}-P_{\theta 0}\right)-\bar{\zeta}\left(P_{\zeta}+\int^{P_{\theta}} \frac{\mathrm{d} P_{\theta}^{\prime}}{q\left(\psi\left(P_{\theta}^{\prime}, P_{\zeta}\right)\right)}\right)-\bar{\xi} \mu
$$

transforms to a new (barred) variable set, related to the original variables as

$$
\begin{aligned}
\bar{P}_{\theta} & =P_{\theta}-P_{\theta 0}, \\
\bar{\theta} & =\theta-\frac{\bar{\zeta}}{q\left(\psi\left(P_{\theta}, P_{\zeta}\right)\right)}, \\
\bar{P}_{\zeta} & =P_{\zeta}+\int^{P_{\theta}} \frac{\mathrm{d} P_{\theta}^{\prime}}{q\left(\psi\left(P_{\theta}^{\prime}, P_{\zeta}\right)\right)}, \\
\zeta & =\bar{\zeta}\left(1+\int^{P_{\theta}} \frac{\partial q^{-1}}{\partial \psi} \frac{\partial \psi\left(P_{\theta}^{\prime}, P_{\zeta}\right)}{\partial P_{\zeta}} \mathrm{d} P_{\theta}^{\prime}\right), \\
\bar{\mu} & =\mu, \\
\bar{\xi} & =\xi .
\end{aligned}
$$

This canonical tranformation is general and can be applied for either axisymmetric or non-axisymmetric equilibrium magnetic fields. The physical meaning of the new canonical variables becomes obvious for a LAR cylindrical equilibrium described by $g=$ $1, I=0, \delta=0$ and $B=1-r \cos \theta$, where $r=\sqrt{2 \psi}$ and the magnetic field is normalized to its on-axis value (White \& Chance 1984). In this case, we have $\partial \psi / \partial P_{\zeta}=0$ and the original canonical momenta, as defined by (2.3), become $P_{\theta}=\psi$ and $P_{\zeta}=\rho_{\|}-\psi_{p}\left(P_{\theta}\right)$, so that $\bar{P}_{\zeta}=\rho_{\|}$and $\bar{\zeta}=\zeta$. The new variables $\left(\bar{P}_{\theta}, \bar{\theta}\right)$ provide the deviation of the GC orbit from a magnetic field line of reference, for which $\theta=\zeta / q(\psi)$, intersecting the poloidal plane $\zeta=0$ at $\left(\psi_{0}, \theta_{0}\right)=\left(P_{\theta 0}, \bar{\theta}\right)$. The new canonical angle $\bar{\theta}$ is directly related to the toroidal precession, defined as $\dot{\zeta}-q \dot{\theta}$. In contrast to previous approaches (Brizard $\&$ Duthoit 2014) where an a posteriori construction of a canonically conjugate pair of variables $\left(\rho_{\|}, s\right)$ for the LAR equilibrium case is necessary, within the context of our formulation, the canonical variables are a priori defined. Moreover, this also holds, not only for the LAR case, but also for an arbitrary equilibrium magnetic field.

For a magnetic field configuration that can be considered as a higher-order perturbation of the LAR equilibrium, according to the standard tokamak ordering, we can write $\psi=P_{\theta}+\epsilon^{2} \hat{\psi}\left(P_{\theta}, P_{\zeta}\right)$, with $\epsilon$ being the inverse aspect ratio and $\hat{\psi}\left(P_{\theta}, P_{\zeta}\right)$ corresponding to corrections due to ellipticity and triangularity (White 2014). Therefore, for the new variable $\bar{\zeta}$ we have

$$
\zeta=\bar{\zeta}\left(1+\epsilon^{2} \int^{P_{\theta}} \frac{\partial q^{-1}}{\partial \hat{\psi}} \frac{\partial \hat{\psi}\left(P_{\theta}^{\prime}, P_{\zeta}\right)}{\partial P_{\zeta}} \mathrm{d} P_{\theta}^{\prime}\right)
$$

and to lowest order $\bar{\zeta}=\zeta$, whereas the constant of the motion can be written as

$$
P_{\zeta}\left(\bar{P}_{\theta}, \bar{P}_{\zeta}\right)=\bar{P}_{\zeta}-\psi_{p}\left(P_{\theta}\right)-\epsilon^{2} \int^{P_{\theta}} \frac{\partial q^{-1}\left(P_{\theta}^{\prime}\right)}{\partial \psi} \psi\left(P_{\theta}^{\prime}, P_{\zeta}\right) \mathrm{d} P_{\theta}^{\prime}
$$

and to lowest order

$$
\bar{P}_{\zeta}=P_{\zeta}+\psi_{p}\left(P_{\theta 0}+\bar{P}_{\theta}\right)
$$


For the case of a smooth $q$ profile we can neglect (locally) higher-order derivatives of $\psi_{p}$ with respect to $\psi=P_{\theta}$, which is equivalent to neglecting the radial variation of the safety factor $q$ (magnetic shear) within a GC drift orbit, and applying a Taylor expansion in $\bar{P}_{\theta} / P_{\theta 0}$ results in

$$
\bar{P}_{\zeta}=P_{\zeta}+\psi_{p}\left(P_{\theta 0}\right)+\psi_{p}^{\prime}\left(P_{\theta 0}\right) \bar{P}_{\theta},
$$

with prime denoting differentiation of $\psi_{p}$ with respect to its argument. Since $P_{\zeta}$ is constant, we can write

$$
\bar{P}_{\zeta}=\rho_{\| 0}+q^{-1}\left(P_{\theta 0}\right) \bar{P}_{\theta},
$$

with $\rho_{\| 0}=P_{\zeta}+\psi_{p}\left(P_{\theta 0}\right)$ depending on both the invariant $P_{\zeta}$ and the flux surface of reference related to $P_{\theta 0}$. This relation imposes a constraint on the two canonical momenta $\bar{P}_{\zeta}$ and $\bar{P}_{\theta}$ due to the axisymmetry of the magnetic field and the corresponding invariance of $P_{\zeta}$. It is worth mentioning that for the case of drift orbits spanning a larger radial distance, we can also include the second-order derivative $\psi_{p}^{\prime \prime}\left(P_{\theta 0}\right)$, related to the magnetic shear $\left(q^{\prime}\right)$, that would result in a quadratic term with respect to $\bar{P}_{\theta}$ in (3.6) and (3.7). As a result, the magnetic shear can modify the GC dynamics and orbital spectrum (Shaing 2015; Albert et al. 2016).

In the new canonical variables the Hamiltonian is given as

$$
\begin{aligned}
H\left(\bar{P}_{\zeta}, \bar{\zeta}, \bar{P}_{\theta}, \bar{\theta} ; \bar{\mu}\right)= & \frac{\bar{P}_{\zeta}^{2}}{2 g^{2}\left(\psi\left(P_{\theta 0}+\bar{P}_{\theta}\right)\right)} B^{2}\left(\psi\left(P_{\theta 0}+\bar{P}_{\theta}\right), \frac{\bar{\zeta}}{q\left(\psi\left(P_{\theta 0}+\bar{P}_{\theta}\right)\right)}+\bar{\theta}\right) \\
& +\bar{\mu} B\left(\psi\left(P_{\theta 0}+\bar{P}_{\theta}\right), \frac{\bar{\zeta}}{q\left(\psi\left(P_{\theta 0}+\bar{P}_{\theta}\right)\right)}+\bar{\theta}\right)+O\left(\epsilon^{2}\right)
\end{aligned}
$$

It is clear that in the new variables there is no cyclic angle, so that neither $\bar{P}_{\theta}$ nor $\bar{P}_{\zeta}$ is a constant of the motion. However, the quantity $P_{\zeta}$ is still a constant of the motion, due to axisymmetry, restricting $\bar{P}_{\theta}$ and $\bar{P}_{\zeta}$ according to (3.7), so that integrability is preserved.

By substituting either of the canonical momenta as a function of the other from (3.7), the Hamiltonian system can be readily described in one degree of freedom either as $H\left(\bar{P}_{\zeta}, \bar{\zeta} ; \rho_{\| 0}, P_{\theta 0} ; \bar{\mu}\right)$ or as $H\left(\bar{P}_{\theta}, \bar{\theta} ; \rho_{\| 0}, P_{\theta 0} ; \bar{\mu}\right)$. In the former case, since the Hamiltonian does not depend on $\bar{P}_{\theta}$, its canonically conjugate angle $\bar{\theta}$ appears as an additive phase constant that can be omitted, whereas the same holds for $\bar{\zeta}$ in the latter case. This duality directly relates the radial and poloidal drift with the toroidal momentum and precession.

The Hamiltonian (3.8) provides a canonical description of the GC motion for a generic axisymmetric magnetic field equilibrium to the lowest order with respect to the standard tokamak ordering and drift orbit deviation from a magnetic field line. Higher-order magnetic field equilibria, with respect to the inverse aspect ratio $\epsilon$, can be considered by keeping higher-order terms in (3.4). The one-degree-of-freedom Hamiltonian $H\left(\bar{P}_{\zeta}, \bar{\zeta} ; \rho_{\| 0}, P_{\theta 0} ; \mu\right)$ can be readily used to calculate the action

$$
J_{\zeta}^{(b, t)}=\frac{1}{2 \pi} \oint \bar{P}_{\zeta}\left(\bar{\zeta} ; E, \bar{\mu}, \rho_{\| 0}, P_{\theta 0}\right) \mathrm{d} \bar{\zeta}
$$

as well as the respective frequencies

$$
\hat{\omega}_{\zeta}^{(b, t)}=\frac{\partial H}{\partial J_{\zeta}^{(b, t)}},
$$

with $E=H$ being the constant energy value of each orbit and the index $(b, t)$ corresponding to the cases of trapped (bounce) or passing (transit) orbits. 
The mixed-variable generating function (Goldstein et al. 2002)

$$
\hat{F}\left(\bar{\zeta}, \bar{\theta}, \bar{\xi} ; J_{\zeta}, J_{\theta}, J_{\xi}\right)=\bar{\xi} J_{\xi}+\bar{\theta} J_{\theta}+\int^{\bar{\zeta}} \bar{P}_{\zeta}\left(\bar{\zeta}^{\prime} ; J_{\zeta}, J_{\theta}, J_{\xi}\right) \mathrm{d} \bar{\zeta}^{\prime}
$$

provides the canonical transformation to action-angle variables also for the remaining canonical variable pairs:

$$
\left.\begin{array}{c}
\left(\bar{P}_{\zeta}, \bar{\zeta}\right) \rightarrow\left(J_{\zeta}, \hat{\zeta}\right), \\
\left(\bar{P}_{\theta}, \bar{\theta}\right) \rightarrow\left(J_{\theta}, \hat{\theta}\right), \\
(\bar{\mu}, \bar{\xi}) \rightarrow\left(J_{\xi}, \hat{\xi}\right) .
\end{array}\right\}
$$

It is worth emphasizing that in the other two pairs of canonical variables, the new momenta (actions) are identical to the old momenta, i.e. $J_{\theta}=\bar{P}_{\theta}$ and $J_{\xi}=\bar{\mu}=\mu$, but the new positions (angles) differ from the old ones, due to the last term of the generating function (3.11) (Kaufman 1972). The transformation to action-angle variables allows for the calculation of the orbital frequencies in the remaining two degrees of freedom. Therefore,

$$
\hat{\omega}_{\theta}=\frac{\partial H}{\partial J_{\theta}}=-\frac{\partial H}{\partial J_{\zeta}} \frac{\partial J_{\zeta}}{\partial J_{\theta}}
$$

and

$$
\hat{\omega}_{\xi}=\frac{\partial H}{\partial J_{\xi}}=-\frac{\partial H}{\partial J_{\zeta}} \frac{\partial J_{\zeta}}{\partial J_{\xi}}
$$

or

$$
\frac{\hat{\omega}_{\theta}}{\hat{\omega}_{\zeta}^{(b, t)}}=-\frac{\partial J_{\zeta}^{(b, t)}}{\partial J_{\theta}}
$$

and

$$
\frac{\hat{\omega}_{\xi}}{\hat{\omega}_{\zeta}^{(b, t)}}=-\frac{\partial J_{\zeta}^{(b, t)}}{\partial J_{\xi}}
$$

The above equations show that the bounce/transit actions $J_{\zeta}^{(b, t)}$ (with a sign change) can be considered as the Hamiltonian functions providing the canonical equations for the other degrees of freedom when time is normalized with respect to the inverse bounce/transit frequency $\hat{\omega}_{\zeta}^{(b, t)}$ (White 2014). Along with the analytical expressions obtained in the next section, these equations provide compact formulas for bounce/transit-averaged dynamics under low-frequency electromagnetic fluctuations in the context of a bounce/gyro-kinetic theory (Brizard 2000; Duthoit, Brizard \& Hahm 2014).

The frequency $\hat{\omega}_{\theta}$ is directly related to the bounce/transit-averaged toroidal drift as shown in the following expression:

$$
\begin{aligned}
\frac{\hat{\omega}_{\theta}}{\hat{\omega}_{\zeta}} & =-\frac{1}{2 \pi} \oint \frac{\partial}{\partial J_{\theta}} \bar{P}_{\zeta}\left(\bar{\zeta} ; E, \bar{\mu}, \rho_{\| 0}, P_{\theta 0}\right) \mathrm{d} \bar{\zeta}=\frac{1}{2 \pi} \oint \frac{\partial \bar{P}_{\zeta}}{\partial E} \frac{\partial E}{\partial J_{\theta}} \mathrm{d} \bar{\zeta} \\
& =\frac{1}{2 \pi} \oint\left(\frac{\mathrm{d} \bar{\zeta}}{\mathrm{d} t}\right)^{-1} \frac{\partial E}{\partial \bar{P}_{\theta}} \mathrm{d} \bar{\zeta}=\frac{1}{2 \pi} \oint\left(\frac{\mathrm{d} \bar{\theta}}{\mathrm{d} t}\right) \mathrm{d} t=\frac{1}{2 \pi}(\Delta \bar{\theta})_{T_{\zeta}=2 \pi / \hat{\omega}_{\zeta}}
\end{aligned}
$$

where $(\Delta \bar{\theta})_{T_{\zeta}=2 \pi / \hat{\omega}_{\zeta}}$ is the variation of $\bar{\theta}=\theta-\zeta / q$ in the time interval of a period $T_{\zeta}=2 \pi / \hat{\omega}_{\zeta}$. Similarly, the frequency $\hat{\omega}_{\xi}$ corresponds to the bounce/transit-averaged 
gyro-frequency (Kaufman 1972):

$$
\frac{\hat{\omega}_{\xi}}{\hat{\omega}_{\zeta}}=\frac{1}{2 \pi}(\Delta \bar{\xi})_{T_{\zeta}=2 \pi / \hat{\omega}_{\zeta}} .
$$

The ZDW approximation, under which the GC is considered as being fixed on a given flux surface $P_{\theta 0}=\psi_{0}$, can be readily obtained by setting $\bar{P}_{\theta}=0$. Moreover, for a relatively small drift width $\bar{P}_{\theta}<<P_{\theta 0}, \psi$ can be Taylor-expanded around $P_{\theta 0}$ and with $\bar{P}_{\theta}$ substituted from (3.7) we have a single-degree-of-freedom Hamiltonian for the canonical variables $\left(\bar{P}_{\zeta}, \bar{\zeta}\right)$.

For a LAR equilibrium, the FDW Hamiltonian is written as

$H_{\mathrm{FDW}}\left(\rho_{\|}, \zeta ; \rho_{\| 0}, \mu ; \psi_{0}\right)=\frac{\rho_{\|}^{2}}{2}\left[1-\sqrt{2 \psi} \cos \left(\frac{\zeta}{q(\psi)}\right)\right]^{2}+\mu\left[1-\sqrt{2 \psi} \cos \left(\frac{\zeta}{q(\psi)}\right)\right]$,

where we have substituted $\bar{P}_{\zeta}=\rho_{\|}$and dropped bar in $\zeta$ for simplicity. It is worth emphasizing that obtaining the Hamiltonian (3.19) is enabled by the utilization of the normalized parallel GC velocity $\rho_{\|}$(normalized to the magnetic field), instead of the regular parallel GC velocity used in previous works (Brizard 2011). Moreover, according to (3.7), $\psi$ is taken as a function of $\rho_{\|}$from

$$
\psi=\psi_{0}+q\left(\psi_{0}\right)\left(\rho_{\|}-\rho_{\| 0}\right),
$$

with $\psi_{0}=P_{\theta 0}$ and $\rho_{\| 0}=P_{\zeta 0}+\psi_{p}\left(\psi_{0}\right)$.

\section{Zero drift width approximation}

We consider the ZDW approximation under which the GC motion is considered fixed on a given flux surface $\psi=\psi_{0}$ and the frequencies of $\theta$ and $\zeta$ are simply related as $\omega_{\zeta}=q\left(\psi_{0}\right) \omega_{\theta}$. The Hamiltonian is written as

$$
H_{\mathrm{ZDW}}\left(\rho_{\|}, \zeta ; \psi_{0}, \mu\right)=\frac{\rho_{\|}^{2}}{2}\left[1-\sqrt{2 \psi_{0}} \cos \left(\frac{\zeta}{q\left(\psi_{0}\right)}\right)\right]^{2}+\mu\left[1-\sqrt{2 \psi_{0}} \cos \left(\frac{\zeta}{q\left(\psi_{0}\right)}\right)\right],
$$

or equivalently as

$$
\begin{aligned}
& H_{\mathrm{ZDW}}\left(\rho_{\|}, \zeta ; \psi_{0}, \mu\right)=\frac{\rho_{\|}^{2}}{2} \\
& \quad+\mu\left\{1-\left[1+\frac{\rho_{\|}^{2}}{\mu}-\frac{\rho_{\|}^{2}}{2 \mu} \sqrt{2 \psi_{0}} \cos \left(\frac{\zeta}{q\left(\psi_{0}\right)}\right)\right] \sqrt{2 \psi_{0}} \cos \left(\frac{\zeta}{q\left(\psi_{0}\right)}\right)\right\} .
\end{aligned}
$$

Further approximations can be based on the relative magnitude of the three terms in the square brackets of the above equation. For $\rho_{\|}^{2} / \mu<<1$, corresponding to particles with large pitch angles $\alpha=\tan ^{-1}\left(v_{\perp} /\left|v_{\|}\right|\right)$, the Hamiltonian is reduced to

$$
H_{\mathrm{ZDW}}^{\prime}\left(\rho_{\|}, \zeta ; \psi_{0}, \mu\right)=\frac{\rho_{\|}^{2}}{2}+\mu\left[1-\sqrt{2 \psi_{0}} \cos \left(\frac{\zeta}{q\left(\psi_{0}\right)}\right)\right]
$$

which resembles the Hamiltonian of a pendulum, with the trapped and passing orbits corresponding to a libration and rotation type of motion (Shaing, Chu \& Sabbagh 2009; 
(a)

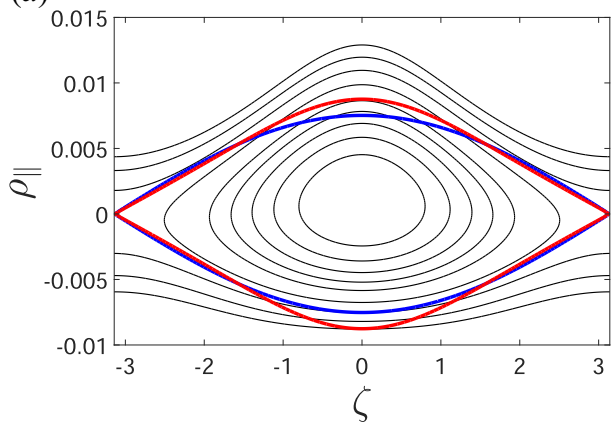

(b)

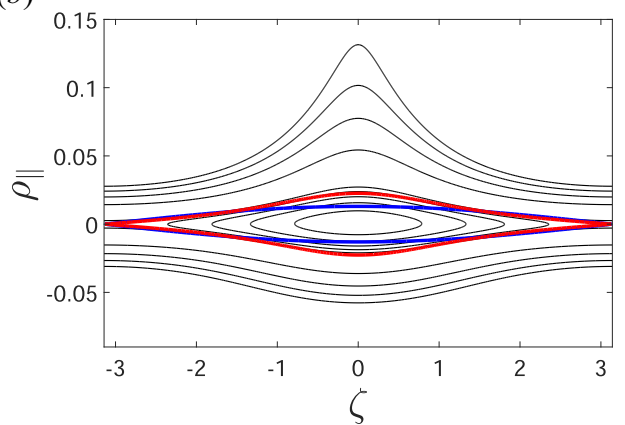

Figure 1. Phase space $\left(\rho_{\|}, \zeta\right)$ of the Hamiltonian $H_{\mathrm{FDW}}$ (3.19) for $q=1, \mu=10^{-4}$ and (a) $\psi_{0}=0.01$ and $(b) \psi_{0}=0.09$. The separatrices between bounce and transit motion according to $H_{\mathrm{ZDW}}(4.1)$ and $H_{\mathrm{ZDW}}^{\prime}(4.3)$ are depicted by blue and red lines, respectively.

Brizard 2011; Brizard \& Duthoit 2014). It is worth mentioning that, under the ZDW approximation, both $H_{\text {ZDW }}$ and $H_{\text {ZDW }}^{\prime}$ scale with $\mu$ when $\rho_{\|}^{2}$ is also divided by $\mu$, which clearly is not the case for the FDW Hamiltonian (3.19).

The above approximations have significant quantitative and qualitative differences. They provide different values for the total energy of a specific orbit and also result in different separatrices between trapped and passing orbits in the phase space, as shown in figure 1 . Therefore, a particle that is described as being trapped according to $H_{\mathrm{ZDW}}^{\prime}$ can be actually passing according to $H_{\text {ZDW }}$ and vice versa. Both ZDW Hamiltonians describe GC orbits that are symmetric with respect to $\rho_{\|}=0$, whereas this is not the case with the FDW Hamiltonian, according to which positive (co-passing) and negative (counter-passing) orbits are not symmetric. These differences are not uniform across the phase space of the system and depend strongly on the pitch angle and the flux surface of reference $\psi_{0}$.

The ZDW Hamiltonians are very useful for obtaining analytical forms for the frequencies of the GC motion and their dependence on the constants of the motion, parametrizing each orbit. These frequencies determine the orbital spectrum of different particle species, including bulk and energetic particles, as well as their resonance conditions with any type of non-axisymmetric perturbations. The latter are crucial for the energy, momentum and particle transport in a toroidal magnetic field configuration.

For both $H_{\text {ZDW }}$ and $H_{\text {ZDW }}^{\prime}$ bounce and transit motion is characterized by $0 \leq k<1$ and $1<k$, respectively, with $k$ being the trapping parameter, defined as

$$
k=\frac{E-\mu(1-r)}{2 \mu r},
$$

where

$$
r=\sqrt{2 \psi_{0}} \text {. }
$$

The bounce/transit frequencies and actions corresponding to $H_{\mathrm{ZDW}}^{\prime}$ are given as follows (appendix A):

$$
\begin{aligned}
& \omega_{b}^{\prime}=\frac{\pi \sqrt{\mu r}}{2 q\left(\psi_{0}\right) K(k)}=\frac{\pi}{2 K(k)} \omega_{b 0}^{\prime}, \\
& \omega_{t}^{\prime}=\frac{\pi \sqrt{\mu r} \sqrt{k}}{q\left(\psi_{0}\right) K\left(k^{-1}\right)}=\frac{\pi \sqrt{k}}{K\left(k^{-1}\right)} \omega_{b 0}^{\prime}
\end{aligned}
$$


and

$$
\begin{aligned}
& J_{b}^{\prime}=\frac{8 q\left(\psi_{0}\right) \sqrt{\mu r}}{\pi}[E(k)+(k-1) K(k)] \\
& J_{t}^{\prime}=\frac{4 q\left(\psi_{0}\right) \sqrt{\mu r}}{\pi} \sqrt{k} E\left(k^{-1}\right),
\end{aligned}
$$

where $K$ and $E$ are the complete elliptic integrals of the first and second kind (Gradshteyn \& Ryzhik 2007), and

$$
\omega_{b 0}^{\prime}=\frac{\pi \sqrt{\mu r}}{2 q\left(\psi_{0}\right) K(0)}=\frac{\sqrt{\mu r}}{q\left(\psi_{0}\right)}
$$

is the frequency of the deeply trapped bounce motion, corresponding to $k=0$. These are the standard analytical expressions for the frequencies and actions obtained from the pendulum-like Hamiltonian $H_{\text {ZDW }}^{\prime}$ (Shaing et al. 2009; Brizard 2011; White 2014).

The respective frequencies and actions for $H_{\mathrm{ZDW}}$ are (appendix A)

$$
\begin{aligned}
& \omega_{b}=\frac{\pi(1-r) \sqrt{\mu r}}{2 q\left(\psi_{0}\right) \Pi(\eta k, k)}=\frac{\pi(1-r)}{2 \Pi(\eta k, k)} \omega_{b 0}^{\prime}, \\
& \omega_{t}=\frac{\pi \sqrt{k}(1-r) \sqrt{\mu r}}{q\left(\psi_{0}\right) \Pi\left(\eta, k^{-1}\right)}=\frac{\pi \sqrt{k}(1-r)}{\Pi\left(\eta, k^{-1}\right)} \omega_{b 0}^{\prime}
\end{aligned}
$$

and

$$
\begin{aligned}
J_{b} & =\frac{8 q\left(\psi_{0}\right) \sqrt{\mu r}}{\pi \eta(1-r)}[(\eta k-1) \Pi(\eta k, k)+K(k)], \\
J_{t} & =\frac{4 q\left(\psi_{0}\right) \sqrt{\mu r}}{\pi \eta(1-r)}\left[\frac{\eta k-1}{\sqrt{k}} \Pi\left(\eta, k^{-1}\right)+\frac{K\left(k^{-1}\right)}{\sqrt{k}}\right],
\end{aligned}
$$

with $\Pi$ being the complete elliptic integral of the third kind (Gradshteyn \& Ryzhik 2007) and

$$
\eta=-\frac{2 r}{1-r}
$$

For a given set of values of the magnetic moment $(\mu)$ and the flux surface $\left(\psi_{0}\right)$, the value of the trapping parameter $(k)$ is determined by the total energy $(E)$ of the particle. The dependence of the bounce and transit frequencies on the energy $E(k)$ according to $H_{\text {ZDW }}^{\prime}$ and $H_{\mathrm{ZDW}}$, as given by (4.6), (4.7) and (4.11), (4.12), respectively, is depicted in figures 2 and 3 . The bounce and transit frequencies $\dot{\theta}$ according to the FDW Hamiltonian in the original canonical variable set, given in (2.4), are also shown. Note that under the ZDW approximation $\dot{\theta}=\dot{\zeta} / q=\dot{\bar{\zeta}} / q=\omega_{b, t} / q=\hat{\omega}_{\zeta} / q$, and we have taken $q=1$ for simplicity. The analytical formula for $\omega_{b}$ (4.11) shows a remarkable agreement with the numerically calculated frequencies based on the FDW Hamiltonian and significantly deviates from the analytical formula for $\omega_{b}^{\prime}$ (4.6) with the deviation being more pronounced for larger values of $\psi_{0}$, as shown in figure 2 . For the transit frequencies, shown in figure 3 , the FDW Hamiltonian describes asymmetric orbits and consequently different frequencies for co-passing $\left(\rho_{\|}>0\right)$ and counter-passing $\left(\rho_{\|}<0\right)$ orbits. The analytical formula for $\omega_{t}$ (4.12) corresponds to an intermediate frequency with respect to the two branches of the numerically calculated FDW frequencies, whereas the analytical formula for $\omega_{t}^{\prime}(4.7)$ tends to follow one of the two branches. 
(a)

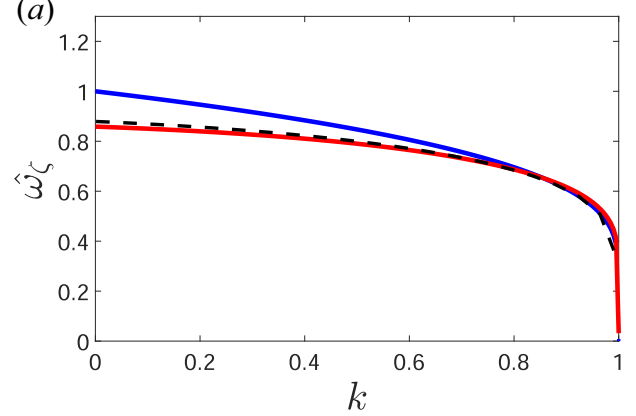

(b)

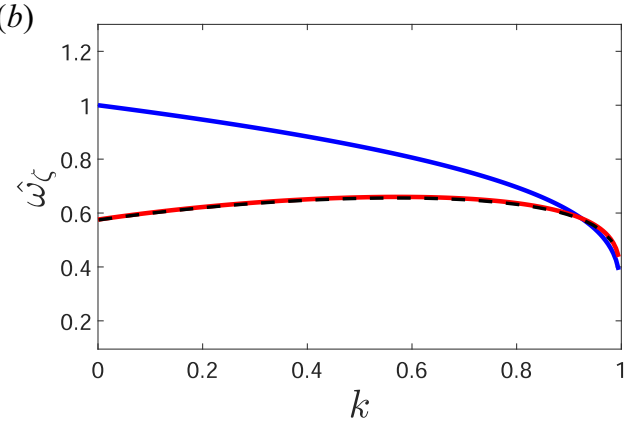

FIGURE 2. Analytically (solid lines) and numerically (dashed lines) calculated bounce frequencies according to $H_{\mathrm{ZDW}}^{\prime}$ (blue line), $H_{\mathrm{ZDW}}$ (red line) and $H(2.4)$ (dashed line) for $q=1$, $\mu=10^{-4}$ and $(a) \psi_{0}=0.01(\eta=-0.33)$ and $(b) \psi_{0}=0.09(\eta=-1.47)$.
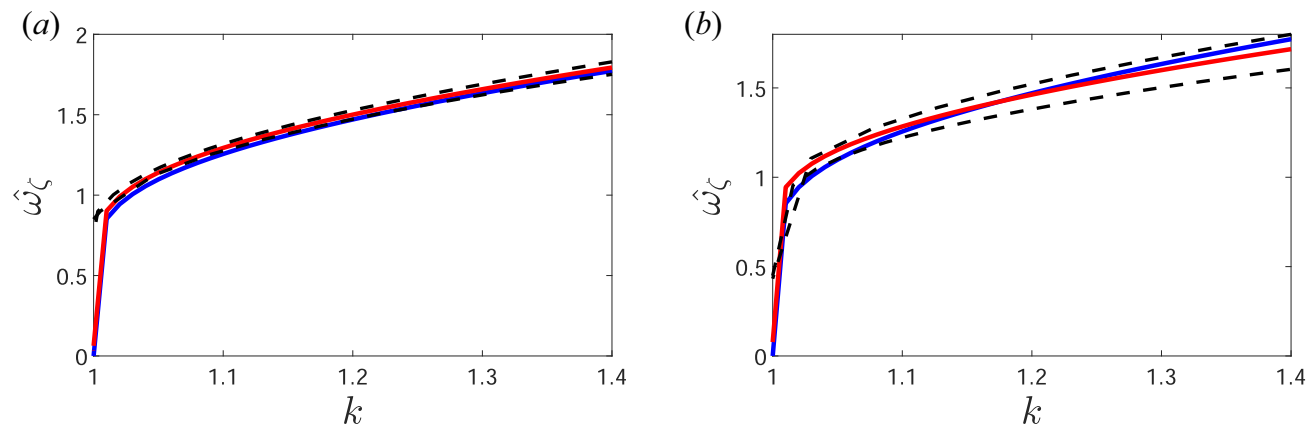

FIGURE 3. Analytically (solid lines) and numerically (dashed lines) calculated transit frequencies according to $H_{\mathrm{ZDW}}^{\prime}$ (blue line), $H_{\mathrm{ZDW}}$ (red line) and $H(2.4)$ (dashed line) for $q=1$, $\mu=10^{-4}$ and $(a) \psi_{0}=0.01(\eta=-0.33)$ and $(b) \psi_{0}=0.09(\eta=-1.47)$.

The two analytical formulas for the bounce frequencies have also a significant qualitative difference. In fact, in contrast to $\omega_{b}^{\prime}, \omega_{b}$ is non-monotonic with respect to $k$ (energy) and, in accordance with FDW numerical calculations, predicts a local maximum of the frequency of the trapped orbits with respect to the energy, indicating the existence of two trapped orbits with different energy but equal frequency, as shown in figure $2(b)$. The location of this local maximum in the space $\left(E(k), \psi_{0}\right)$ is depicted in figure 4 . The non-monotonic dependence of the frequency on the particle energy has important implications for particle and momentum transport in the presence of non-axisymmetric perturbations that break the integrability of the system. In such cases, the locations of the phase space regions where orbits are significantly modified due to perturbations are determined by resonance conditions. The non-monotonicity suggests that the same resonance can modify two regions of the phase space leading to extended transport under resonance overlap conditions. Moreover, the existence of energy values where the derivative of the frequency with respect to energy is zero can be related to an intrinsic degeneracy condition which is crucial for the effect of perturbations (Lichtenberg \& Lieberman 1992).

The bounce-averaged toroidal precession and gyration frequencies can be analytically caclulated according to (3.15) and (3.16) with the utilization of either $J_{b}$ (4.13), $\omega_{b}$ (4.11) or $J_{b}^{\prime}$ (4.8), $\omega_{b}^{\prime}$ (4.6). The resulting expressions are too lengthy to be given here; their dependence on energy $(k)$ is depicted in figures 5 and 6 . A sign reversal in the 
(a)

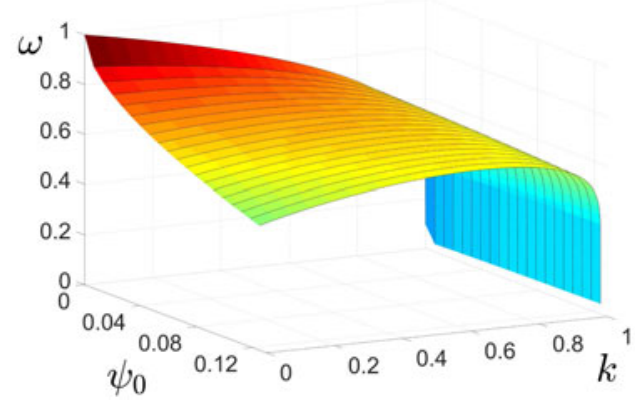

(c)

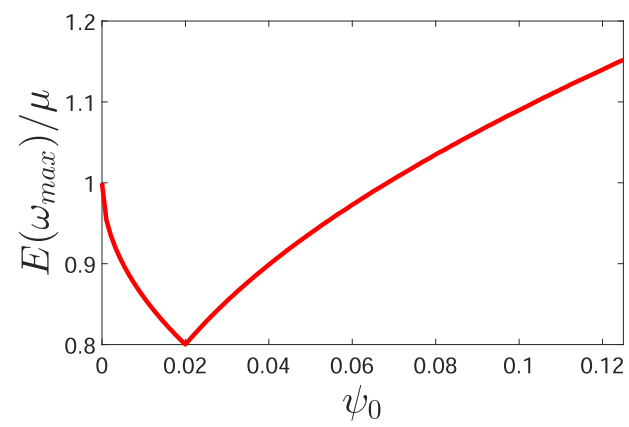

(b)

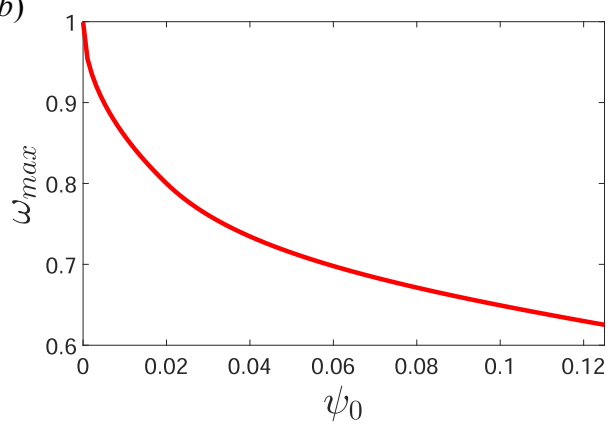

(d)

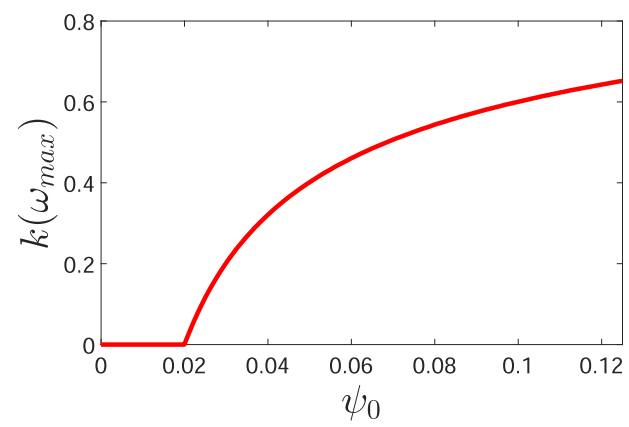

FIGURE 4. (a) Bounce frequency $\omega_{b}$ dependence on energy $(k)$ and $\psi_{0}$. (b) Maximum bounce frequency as a function of $\psi_{0}$. $(c, d)$ Energy and $k$ corresponding to the maximum bounce frequency; for small $\psi_{0}$, the maximum frequency corresponds to the deeply trapped $k \simeq 0$ particles.

bounce-averaged toroidal precession frequency is shown in figure 5. The analytical results based on $H_{\text {ZDW }}$ show a remarkable agreement with the numerical results based on the FDW Hamiltonian, where the expression (3.17) has been utilized. The differences between analytical results based on $H_{\mathrm{ZDW}}$ and $H_{\mathrm{ZDW}}^{\prime}$ significantly differ for larger values of $\psi_{0}$. These differences imply different phase space regions where resonant interactions with low-frequency electromagnetic perturbations take place (Duthoit et al. 2014). Similar differences are also shown for the bounce-averaged gyration frequencies in figure 6, determining the conditions for resonant interactions with high-frequency waves.

\section{Summary and conclusions}

The GC motion in an axisymmetric magnetic field is analysed under a Hamiltonian formulation in canonical variables. The ZDW approximation has been described in the context of the canonical formulation through a canonical transformation to variables measuring the deviation of the GC from a magnetic field line of reference. The latter allows for the expression of the system in action-angle variables and the systematic dynamical reduction to lower-dimensional phase spaces, depending on the specific physical problem under consideration, such as low- or high-frequency perturbations.

A novel ZDW Hamiltonian has been obtained along with compact analytical formulas for all the GC orbital frequencies, namely bounce/transit frequency and bounce-averaged toroidal precession and gyration frequency. The analytical results significantly differ 

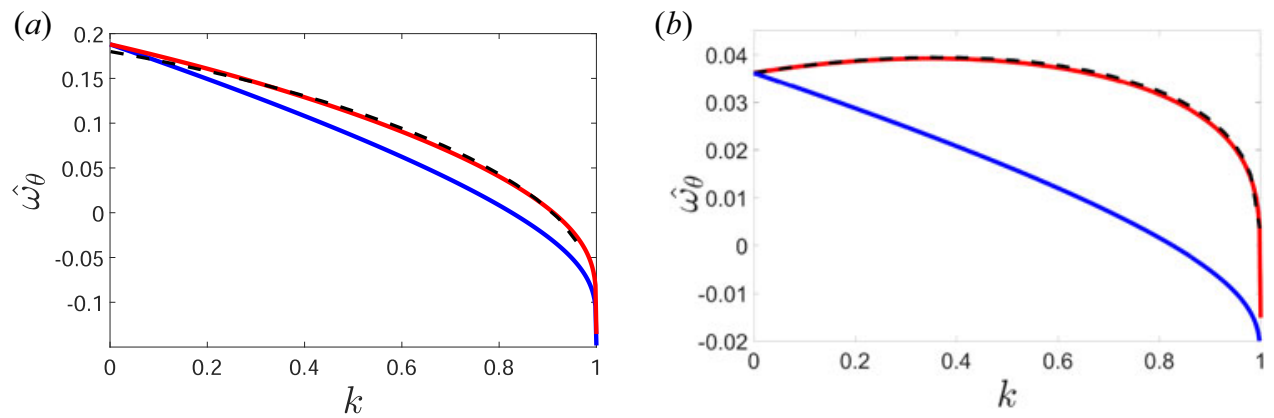

FIGURE 5. Analytically (solid lines) and numerically (dashed lines) calculated bounce-averaged toroidal precession frequencies according to $H_{\text {ZDW }}^{\prime}$ (blue line), $H_{\text {ZDW }}$ (red line) and $H(2.4)$ (dashed line) for $q=1, \mu=10^{-4}$ and $(a) \psi_{0}=0.01(\eta=-0.33)$ and (b) $\psi_{0}=0.09(\eta=-1.47)$.

(a)

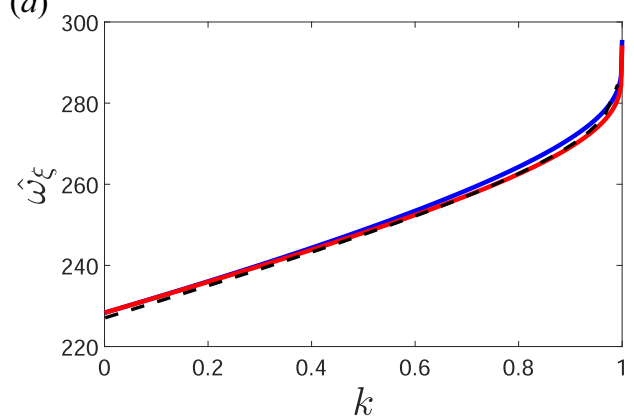

(b)

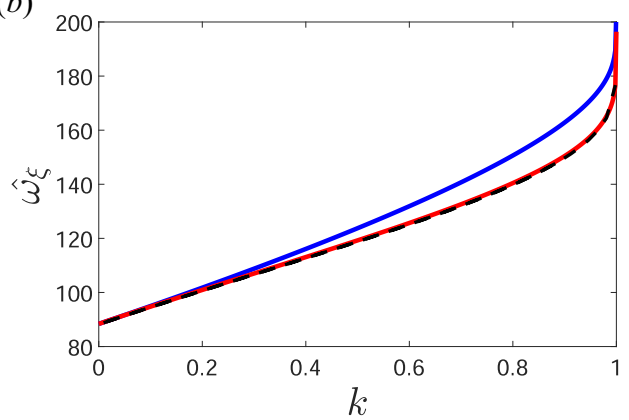

FIGURE 6. Analytically (solid lines) and numerically (dashed lines) calculated bounce-averaged gyro-frequencies according to $H_{\text {ZDW }}^{\prime}$ (blue line), $H_{\text {ZDW }}$ (red line) and $H$ (2.4) (dashed line) for $q=1, \mu=10^{-4}$ and $(a) \psi_{0}=0.01(\eta=-0.33)$ and $(b) \psi_{0}=0.09$ $(\eta=-1.47)$.

from those corresponding to the widely used pendulum-like Hamiltonian and show a remarkable agreement with numerically calculated frequencies for the FDW Hamiltonian.

The knowledge of the orbital frequencies is crucial for determining the resonance conditions under particle interaction with non-axisymmetric perturbations that affect energy, momentum and particle transport in toroidal plasma configurations. Moreover, the transformation to action-angle variables allows for the application of standard canonical perturbation methods as well as the systematic dynamical reduction and the formulation of a bounce gyro-kinetic description.

\section{Acknowledgements}

G.A. and Y.K. acknowledge useful discussions with P. A. Zestanakis. This work has been carried out within the framework of the EUROfusion Consortium and has received funding from the Euratom research and training programme 2014-2018 and 2019-2020 under grant agreement no. 633053 as well as from the National Programme for Controlled Thermonuclear Fusion, Hellenic Republic. The views and opinions expressed herein do not necessarily reflect those of the European Commission. 
Editor Christos Tsironis thanks the referees for their advice in evaluating this article.

\section{Declaration of interests}

The authors report no conflict of interest.

\section{Appendix A. Action-angle variables}

The ZDW Hamiltonians $H_{\mathrm{ZDW}}$ and $H_{\mathrm{ZDW}}^{\prime}$ given in (4.1) and (4.3) can be written as

$$
H\left(\rho_{\|}, \zeta ; \psi_{0}, \mu ; \sigma\right)=\frac{\rho_{\|}^{2}}{2}\left[1-\sigma r \cos \left(\frac{\zeta}{q\left(\psi_{0}\right)}\right)\right]^{2}+\mu\left[1-r \cos \left(\frac{\zeta}{q\left(\psi_{0}\right)}\right)\right]=E
$$

with $\sigma=1$ and $\sigma=0$, respectively. The equation $H=E$, where $E$ is the constant energy, can be solved with respect to $\rho_{\|}$as follows:

$$
\rho_{\|}^{2}=2 \frac{(E-\mu)+\mu r \cos \left(\frac{\zeta}{q\left(\psi_{0}\right)}\right)}{\left[1-\sigma r \cos \left(\frac{\zeta}{q\left(\psi_{0}\right)}\right)\right]^{2}} .
$$

The corresponding action variables are defined as

$$
J=\oint \rho_{\|} \mathrm{d} \zeta=\oint \frac{\sqrt{2[(E-\mu)+\mu r \cos (\zeta / q)]}}{1-\sigma r \cos (\zeta / q)} \mathrm{d} \zeta
$$

and, by setting $\zeta / 2 q \equiv \chi$ and using $\cos 2 \chi=1-2 \sin ^{2} \chi$, the action variables are given as

$$
J=\frac{2^{l+1} q\left(\psi_{0}\right) \sqrt{k} \sqrt{\mu r}}{\pi(1-\sigma r)} \int_{\chi_{\min }}^{\chi_{\max }} \frac{\sqrt{1-k^{-1} \sin ^{2} \chi}}{1-\eta \sin ^{2} \chi} \mathrm{d} \chi
$$

with $k=[E-\mu(1-r)] / 2 \mu r, \quad \eta=-2 \sigma r /(1-\sigma r)$ and $\chi_{\min , \max }=\sin ^{-1}(\mp \sqrt{k})$ for trapped orbits (bounce motion, $0 \leq k<1, l=1$ ) and $\chi_{\min , \max }=\mp \pi / 2$ for passing orbits (transit motion $k>1, l=0$ ).

The equation of motion $\mathrm{d} \zeta / \mathrm{d} t=\partial H / \partial \rho_{\|}$can be written as

$$
\mathrm{d} t= \pm \frac{q\left(\psi_{0}\right)}{(1-\sigma r) \sqrt{k} \sqrt{\mu r}} \frac{\mathrm{d} \chi}{\left(1-\eta \sin ^{2} \chi\right) \sqrt{1-k^{-1} \sin ^{2} \chi}} .
$$

The integration of the above relation over a closed orbit provides the period of the bounce and the transit motion as

$$
T=\frac{2^{l} q\left(\psi_{0}\right)}{(1-\sigma r) \sqrt{k} \sqrt{\mu r}} \int_{\chi_{\min }}^{\chi_{\max }} \frac{\mathrm{d} \chi}{\left(1-\eta \sin ^{2} \chi\right) \sqrt{1-k^{-1} \sin ^{2} \chi}},
$$

with the respective frequency given as $\omega=2 \pi / T$.

The resulting expressions for the bounce and transit frequencies and actions for the Hamiltonian $H_{\mathrm{ZDW}}^{\prime}$ are given in terms of complete elliptic integrals of the first and second kind (Gradshteyn \& Ryzhik 2007) as in (4.6), (4.7) and (4.8), (4.9). For the Hamiltonian $H_{\text {ZDW }}$, the corresponding expressions are given in terms of complete elliptic integrals of the third kind (Gradshteyn \& Ryzhik 2007) as in (4.11), (4.12) and (4.13), (4.14). 
The equation of motion (A 5) can also be used for the calculation of the transformation to the angle variable $\hat{\zeta}$ in terms of incomplete elliptic integrals as follows:

$$
\hat{\zeta} \equiv \omega t=\frac{q\left(\psi_{0}\right) \omega(k, \mu, r ; \sigma)}{(1-\sigma r) \sqrt{k} \sqrt{\mu r}} \int_{\chi_{\min }}^{\chi} \frac{\mathrm{d} \chi^{\prime}}{\left(1-\eta \sin ^{2} \chi^{\prime}\right) \sqrt{1-k^{-1} \sin ^{2} \chi^{\prime}}},
$$

where we have taken $\hat{\zeta}=0$ when $\chi=\chi_{\min }$. Note that this relation defines the transformation from $\zeta=2 q\left(\psi_{0}\right) \chi$ to $\hat{\zeta}$ in an implicit form. For the case of the Hamiltonian $H_{\text {ZDW }}^{\prime}$ corresponding to $\sigma=0$, the relation can be inverted with the use of Jacobi elliptic functions, whereas for the case of $H_{\text {ZDW }}$ corresponding to $\sigma=1$, the inversion of the relation, to the best of our knowledge, cannot be expressed in a convenient form (Gradshteyn \& Ryzhik 2007).

\section{REFERENCES}

Albert, C. G., Heyn, M. F., Kapper, G., Kasilov, S. V., Kernbichler, W. \& Martitsch, A. F. 2016 Evaluation of toroidal torque by non-resonant agnetic perturbations in tokamaks for resonant transport regimes using a hamiltonian approach. Phys. Plasmas 23, 082515.

Bierwage, A. \& Shinohara, K. 2016 Orbit-based analysis of nonlinear energetic ion dynamics in tokamaks. I. Effective mode number profile and resonant frequency tracking. Phys. Plasmas 23, 042511.

BRIZARD, A. J. 2000 Nonlinear bounce-gyrocenter hamiltonian dynamics in general magnetic field geometry. Phys. Plasmas 7, 3238-3246.

BRIZARD, A. J. 2011 Compact formulas for guiding-center orbits in axisymmetric tokamak geometry. Phys. Plasmas 18, 022508.

BRiZARD, A. J. \& Duthoit, F. X. 2014 Canonical transformation for trapped/passing guiding-center orbits in axisymmetric tokamak geometry. Phys. Plasmas 21, 052509.

Duthoit, F. X., BRIZARD, A. J. \& HAHM, T. S. 2014 Compact formulas for bounce/transit averaging in axisymmetric tokamak geometry. Phys. Plasmas 21, 122510.

Freidberg, J. P. 2007 Plasma Physics and Fusion Energy. Cambridge University Press.

Goldstein, H., Poole, C. P. \& SAFKo, J. L. 2002 Classical Mechanics, 3rd edn. Addison-Wesley.

Gradshteyn, I. S. \& Ryzhik, I. M. 2007 Table of Integrals, Series, and Products, 7th edn. Academic Press.

Kaufman, A. N. 1972 Quasilinear diffusion of an axisymmetric toroidal plasma. Phys. Fluids 15, 1063-1069.

Kominis, Y. 2008 Nonlinear theory of cyclotron resonant wave-particle interactions: analytical results beyond the quasilinear approximation. Phys. Rev. E 77, 016404.

Kominis, Y., RAM, A. K. \& HizAnidis, K. 2008 Quasilinear theory of electron transport by radio frequency waves and nonaxisymmetric perturbations in toroidal plasmas. Phys. Plasmas 15, 122501.

Kominis, Y., RAM, A. K. \& Hizanidis, K. 2010 Kinetic theory for distribution functions of wave-particle interactions in plasmas. Phys. Rev. Lett. 104, 235001.

Lichtenberg, A. J. \& Lieberman, M. A. 1992 Regular and Chaotic Dynamics, 2nd edn. Springer.

Littlejohn, R. G. 1983 Variational principles of guiding center motion. J. Plasma Phys. 29, 111-125.

Littlejohn, R. G. 1985 Differential forms and canonical variables for drift motion in toroidal geometry. Phys. Fluids 28, 2015-2016.

Petrov, Y. V. \& Harvey, R. W. 2016 A fully-neoclassical finite-orbit-width version of the cql3d fokker-planck code. Plasma Phys. Control. Fusion 58, 115001.

Podesta, M., Gorelenkova, M. \& White, R. B. 2014 A reduced fast ion transport model for the tokamak transport code transp. Plasma Phys. Control. Fusion 56, 055003.

SHAING, K. C. 2015 Superbanana and superbanana plateau transport in finite aspect ratio tokamaks with broken symmetry. J. Plasma Phys. 81, 905810203. 
Shaing, K. C., Chu, M. S. \& Sabbagh, S. A. 2009 Eulerian approach to bounce-transit and drift resonance and neoclassical toroidal plasma viscosity in tokamaks. Plasma Phys. Control. Fusion 51, 075015 .

Stagner, L. \& Heidbrink, W. W. 2017 Action-angle formulation of generalized, orbit-based, fast-ion diagnostic weight functions. Phys. Plasmas 24, 092505.

Tholerus, E., Johnson, T. \& Hellsten, T. 2017 Foxtail: Modeling the nonlinear interaction between alfven eigenmodes and energetic particles in tokamaks. Comput. Phys. Commun. 214, 39-51.

White, R. B. 2011 Modification of particle distributions by mhd instabilities ii. Plasma Phys. Control. Fusion 53, 085018.

White, R. B. 2012 Modification of particle distributions by mhd instabilities i. Commun. Nonlinear Sci. Numer. Simul. 17, 2200-2214.

White, R. B. 2014 The Theory of Toroidally Confined Plasmas, 3rd edn. Imperial College Press.

White, R. B. \& ChAnCE, M. S. 1984 Hamiltonian guiding center drift orbit calculation for plasmas of arbitrary cross section. Phys. Fluids 27, 2455-2467.

Zestanakis, P. A., Kominis, Y., Anastassiou, G. \& Hizanidis, K. 2016 Orbital spectrum analysis of non-axisymmetric perturbations of the guiding-center particle motion in axisymmetric equilibria. Phys. Plasmas 23, 032507. 